\title{
A simple synthesis of 2-Acetyl-5-hydroxy-1,2,3,4 tetrahydronaphthalene
}

\author{
William J. Vera ${ }^{*}$ and Ajoy K. Banerjee* \\ IVIC, Centro de Química, Apartado 21827, Caracas 1020-A Venezuela \\ E-mail: abanerje@ivic.ve
}

Dedicated to Professor Atta-ur-Rahman on the occasion of his $65^{\text {th }}$ Birthday

\begin{abstract}
5-Methoxy-1-tetralone $\mathbf{1}$ has been converted into 2-Acetyl-5-methoxy-1,2,3,4tetrahydronaphthalene 5 by acylation, hydrogenation and oxidation respectively. Demethylation of 5 with boron tribromide afforded the title compound 6 .
\end{abstract}

Keywords: acylation, demethylation, hydrogenolysis, oxidation

\section{Introduction}

Several years ago Rao and collaborators reported ${ }^{1,2}$ the synthesis of the tittle compound 6 and its transformation into 7,9,11-trideoxydaunomycinone 7 which can be smoothly converted into aglycones of the antitumor anthracycline antibiotic $( \pm)$-11-deoxydaunomycinone. The reported synthesis of $\mathbf{6}$ involves nine steps. In this paper we describe a short and simple synthesis of $\mathbf{6}$ from the commercially available 5-methoxy-1-tetralone 1.

Acylation $^{3}$ of tetralone $\mathbf{1}$ with ethyl acetate yielded a crystalline solid whose spectroscopic date outlined in the experimental section suggested the exclusive formation of C-acetyl enol 2. This on hydrogenation with $\mathrm{PtO}_{2}$ in ethanol and acetic acid at room temperature and atmospheric pressure yielded the compound $3(88 \%)$ due to hydrogenolysis as well as hydrogenation. In addition, another compound 4 (12\%) was obtained whose formation indicated that the compound 3 partially underwent hydrogenolysis. Oxidation of 3 with Sarett reagent ${ }^{4}$ afforded ketone 5 which on demethylation with boron tribromide in dichloromethane produced the target compound 6 in $46 \%$ yield. Its m.p and spectral data (ir and nmr) matched closely with those of the data reported ${ }^{2}$. 

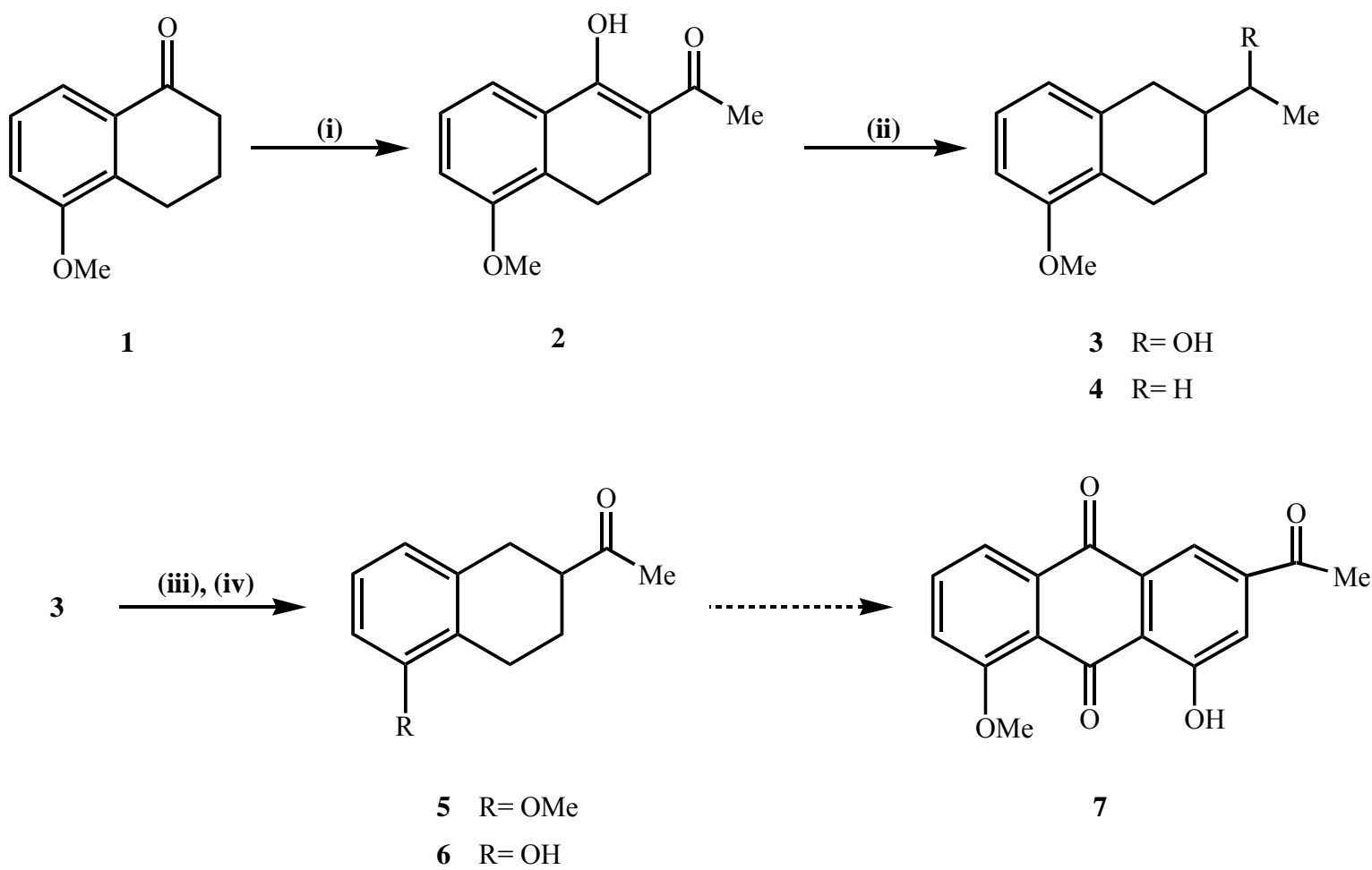

Reagents: (i) AcOEt, $\mathrm{C}_{6} \mathrm{H}_{6}, \mathrm{DMSO}, \mathrm{NaOMe}$, (ii) $\mathrm{H}_{2}, \mathrm{PtO}_{2}, \mathrm{AcOH}, \mathrm{EtOH}$, (iii) $\mathrm{CrO}_{3}, \mathrm{Py}$, (iv) $\mathrm{BBr}_{3}, \mathrm{CH}_{2} \mathrm{Cl}_{2}$

In conclusion, a four step synthesis of compound $\mathbf{6}$ has been accomplished and we believe that the present method can be utilized for the synthesis of 7 in quantities sufficient to accomplish the total synthesis of $( \pm)$-11-deoxydaunomycinone.

\section{Experimental Section}

General Procedures. Unless otherwise stated, IR spectra were taken on Nicolet FT and NMR spectra recorded on a Bruker AM-300 spectrometer were taken in $\mathrm{CDCl}_{3}$. Mass spectra were carried on Dupont 21-492B. Column chromatography was carried out on silica gel 60 (Merck) and TLC plates were coated with silica gel and the spots were located by exposing to UV light. Microanalyses were carried out in the Chemistry Department, IVIC, Caracas.

2-Acetyl-5-methoxy-1-hydroxy-3,4-dihydronaphthalene (2). To a solution of 5-methoxy-1tetralone $1(2.01 \mathrm{~g}, 11.4 \mathrm{mmol})$ in a mixture of benzene $(46.3 \mathrm{~mL})$, ethyl acetate $(11.6 \mathrm{~mL})$ and dimethylsulfoxide $(11.6 \mathrm{~mL})$, warmed at $50^{\circ} \mathrm{C}$, was added a solution of sodium methoxide (1.01 $\mathrm{g} \mathrm{Na}, 3 \mathrm{~mL}$ methanol). The mixture was refluxed for $1.5 \mathrm{~h}$, cooled and poured into ice water. The organic phase was washed with a solution of sodium hydroxide $(5 \%)$. The combined extracts were acidified with conc. hydrochloric acid and then again extracted with dichloromethane. The combined organic extracts were washed, dried and evaporated. The resulting solid material on crystallization with hexane-ether (9:1) afforded C-acetyl enol 2 (1.91 
g, 77\%), m.p. $50-53^{\circ} \mathrm{C}$; I.R. $\mathrm{U}_{\max }\left(\mathrm{cm}^{-1}\right): 1672(\mathrm{CO})$ and $1582(\mathrm{C}=\mathrm{C}) ; \mathrm{MS}(\mathrm{m} / \mathrm{z}): 218\left(\mathrm{M}^{+}\right.$, 100\%), $203\left(\mathrm{M}^{+}-\mathrm{Me}\right)(7 \%), 175\left(\mathrm{M}^{+}-\mathrm{MeCO}\right)(15 \%) ;{ }^{1} \mathrm{H} \delta_{(\mathrm{ppm})}: 16.38(\mathrm{~s}, 1 \mathrm{H}, \mathrm{OH}) 7.55(\mathrm{dd}, 1 \mathrm{H}$, $\mathrm{J}=1.04 \mathrm{~Hz} ; \mathrm{J}=8 \mathrm{~Hz}$ ) (ArH at C-8), $7.24(\mathrm{t}, 1 \mathrm{H}, \mathrm{J}=8 \mathrm{~Hz})(\mathrm{ArH}$ at $\mathrm{C}-7), 6.95(\mathrm{dd}, 1 \mathrm{H}, \mathrm{J}=8 \mathrm{~Hz}$, $\mathrm{J}=1 \mathrm{~Hz})\left(\mathrm{ArH}\right.$ at C-6), $3.82(\mathrm{~s}, 3 \mathrm{H}, \mathrm{OMe}), 2.83(\mathrm{t}, 2 \mathrm{H}, \mathrm{J}=7 \mathrm{~Hz})\left(\mathrm{CH}_{2}\right.$ at $\left.\mathrm{C}-4\right), 2.55(\mathrm{t}, 2 \mathrm{H}, \mathrm{J}=7 \mathrm{~Hz})$, $\left(\mathrm{CH}_{2}\right.$ at $\left.\mathrm{C}-3\right)$, and $2.20(\mathrm{~s}, 3 \mathrm{H}, \mathrm{COMe}) ;{ }^{13} \mathrm{C} \delta_{(\mathrm{ppm})}: 193(\mathrm{C}-12), 176(\mathrm{C}-1), 155(\mathrm{C}-5), 132(\mathrm{C}-9)$, 129 (C-10), 126 (C-7), 118 (C-8), 113 (C-2), 105 (C-6), 55 (C-11), 23 (C-3), 22 (C-4), 20 (C13). (Found: $\mathrm{C}, 71.91 ; \mathrm{H}, 6.65 ; \mathrm{C}_{13} \mathrm{H}_{14} \mathrm{O}_{3}$ requires $\mathrm{C}, 71.54 ; \mathrm{H}, 6.47$ ).

2-Ethyl-5-methoxy-1,2,3,4-tetrahydronaphthalene (4) and 2-hydroxymethyl-5-methoxy1,2,3,4-tetrahydronaphthalene (3). A solution of compound 2 (1.22 g, $5.6 \mathrm{mmol})$ in ethanol (40 $\mathrm{mL})$ and acetic acid $(15 \mathrm{~mL})$ was hydrogenated in presence of $\mathrm{PtO}_{2}(161 \mathrm{mg}, 0.71 \mathrm{mmol})$ under atmospheric pressure of hydrogen for $6 \mathrm{~h}$. Evaporation of the solvent gave an oil which was dissolved in dichloromethane. The organic extract was washed with a solution of sodium bicarbonate $(5 \%)$ and then with brine, dried and evaporated. The resulting oil was chromatographed over silica gel with hexane:ether (4:1) to give 2-ethyl-5-methoxy-1,2,3,4tetrahydronaphthalene $4(130 \mathrm{mg}, 12 \%)$; $\mathrm{MS}(\mathrm{m} / \mathrm{z}): 190\left(\mathrm{M}^{+}\right)(100 \%)$ and $161\left(\mathrm{M}^{+}-\mathrm{C}_{2} \mathrm{H}_{5}\right)$ $(53 \%) ;{ }^{1} \mathrm{H} \delta_{(\mathrm{ppm})}: 7.09$ (t, 1H, J=8 Hz) (ArH at C-7), 6.72 (d, 1H, J=8 Hz) (ArH at C-6), 6.66 (d, $1 \mathrm{H}, \mathrm{J}=8 \mathrm{~Hz})\left(\mathrm{ArH}\right.$ at C-8), $3.83(\mathrm{~s}, 3 \mathrm{H}, \mathrm{OMe}), 2.85(\mathrm{~m}, 2 \mathrm{H})\left(\mathrm{CH}_{2}\right.$ at $\left.\mathrm{C}-4\right), 2.48(\mathrm{~m}, 2 \mathrm{H})\left(\mathrm{CH}_{2}\right.$ at $\mathrm{C}-1), 2.01(\mathrm{~m}, 1 \mathrm{H})(\mathrm{CH}$ at $\mathrm{C}-2), 1.37(\mathrm{~m}, 4 \mathrm{H})\left(2 \mathrm{xCH}_{2}\right.$ at $\mathrm{C}-3$ and $\left.\mathrm{C}-11\right), 1.01(\mathrm{t}, 3 \mathrm{H}, \mathrm{J}=7 \mathrm{~Hz})$ (Me at C12). (Found: $\mathrm{C}, 82.34 ; \mathrm{H}, 9.69 ; \mathrm{C}_{13} \mathrm{H}_{18} \mathrm{O}$ requires $\mathrm{C}, 82.06 ; \mathrm{H}, 9.54$ ).

Further elution with hexane:ether (7:3) afforded 2-hydroxymethyl-5-methoxy 1,2,3,4tetrahydronaphthalene 3 (1.01 g, 88\%); I.R. $\cup_{\max }\left(\mathrm{cm}^{-1}\right): 3377(\mathrm{OH}) ; \mathrm{MS}(\mathrm{m} / \mathrm{z}): 206\left(\mathrm{M}^{+}\right)(90 \%)$, $188\left(\mathrm{M}^{+} \cdot-\mathrm{H}_{2} \mathrm{O}\right)(50 \%), 173\left(\mathrm{M}^{+} \cdot-\mathrm{Me}-\mathrm{H}_{2} \mathrm{O}\right)(32 \%), 160\left(\mathrm{M}^{+}-\mathrm{MeCHOH}\right)(52 \%) ;{ }^{1} \mathrm{H} \delta_{(\mathrm{ppm})}: 7.15$ $(\mathrm{t}, 1 \mathrm{H}, \mathrm{J}=8 \mathrm{~Hz})(\mathrm{ArH}$ at C-7), $6.78(\mathrm{~d}, 1 \mathrm{H}, \mathrm{J}=8 \mathrm{~Hz})(\mathrm{ArH}$ at C-6), $6.72(\mathrm{~d}, 1 \mathrm{H}, \mathrm{J}=8 \mathrm{~Hz}$ ) (ArH at C8), $3.87(\mathrm{~s}, 3 \mathrm{H}, \mathrm{OMe}), 3.71(\mathrm{~m}, 1 \mathrm{H}, \mathrm{CHOH}), 2.91(\mathrm{~m}, 2 \mathrm{H})\left(\mathrm{CH}_{2}\right.$ at $\left.\mathrm{C}-4\right), 2.61(\mathrm{~m}, 2 \mathrm{H})\left(\mathrm{CH}_{2}\right.$ at $\mathrm{C}-$ 1), $2.26(\mathrm{~m}, 1 \mathrm{H})(\mathrm{CH}$ at $\mathrm{C}-2), 1.46(\mathrm{~m}, 2 \mathrm{H})\left(\mathrm{CH}_{2}\right.$ at $\left.\mathrm{C}-3\right), 1.31(\mathrm{~d}, 3 \mathrm{H}, \mathrm{J}=6 \mathrm{~Hz}, \mathrm{Me}) ;{ }^{13} \mathrm{C} \delta_{(\mathrm{ppm})}$ : 156.9 (C-5), 137.4 (C-9), 125.6 (C-7), 125.3 (C-10), 121.3 (C-8), 106.5 (C-6), 70.9 (C-11), 54.8 (C-13), 41.1 (C-2), 31.7 (C-1), 24.4 (C-3), 22.9 (C-4), 20.2 (C-12). (Found: C, 75.95; H, 8.98; $\mathrm{C}_{13} \mathrm{H}_{18} \mathrm{O}_{2}$ requires $\left.\mathrm{C}, 75.69 ; \mathrm{H}, 8.80\right)$.

2-Acetyl-5-methoxy-1,2,3,4-tetrahydronaphthalene (5). A solution of compound 3 (1g, 4.8 $\mathrm{mmol})$ in pyridine $(10 \mathrm{~mL})$ was added to the Sarett reagent, prepared from dry chromium trioxide $(1.95 \mathrm{~g}, 19.5 \mathrm{mmol})$ and dry pyridine $(25 \mathrm{~mL})$, at $\mathrm{O}^{\circ} \mathrm{C}$. The mixture was left to stand at room temperature for $20 \mathrm{~h}$, diluted with water and extracted with ether. The ether extract was dried, evaporated and chromatographed over silica gel to give the ketone 5 (594 mg, 60\%); I.R. $\mathrm{U}_{\max }\left(\mathrm{cm}^{-1}\right): 1708(\mathrm{CO})$; MS (m/z): $204\left(\mathrm{M}^{+}\right)(100 \%), 189\left(\mathrm{M}^{+}-\mathrm{Me}\right)(13 \%), 161\left(\mathrm{M}^{+}-\mathrm{COMe}\right)$ $(49 \%) ;{ }^{1} \mathrm{H} \delta_{(\mathrm{ppm})}: 7.08$ (t, 1H, J=8 Hz) (ArH at C-7), $6.71(\mathrm{~d}, 1 \mathrm{H}, \mathrm{J}=8 \mathrm{~Hz})(\mathrm{ArH}$ at C-6), 6.64 (d, $1 \mathrm{H}, \mathrm{J}=8 \mathrm{~Hz}$ ) (ArH at C-8), $3.78(\mathrm{~s}, 3 \mathrm{H}, \mathrm{OMe}), 2.74(\mathrm{~m}, 5 \mathrm{H}), 2.21(\mathrm{~s}, 3 \mathrm{H}, \mathrm{COMe}), 1.62(\mathrm{~m}, 2 \mathrm{H})$; ${ }^{13} \mathrm{C} \delta_{(\mathrm{ppm})}: 210.6(\mathrm{C}-13), 156.8$ (C-5), 136 (C-9), 125.8 (C-7), 124.2 (C-10), 120.9 (C-8), 106.7 (C-6), 54.7(C-12), 47.2 (C-2), 30.7 (C-1), 27.7 (C-3), 24.7 (C-11), 22.5 (C-4) (Found: C,76.79; $\mathrm{H}, 8.06 ; \mathrm{C}_{13} \mathrm{H}_{16} \mathrm{O}_{2}$ requires $\left.\mathrm{C}, 76.44 ; \mathrm{H}, 7.90\right)$. 
2-Acetyl-5-hydroxy-1,2,3,4-tetrahydronaphthalene (6). To a solution of compound 5 (300 mg, $1.5 \mathrm{mmol})$ in dichloromethane $(3 \mathrm{~mL})$ cooled at $-10^{\circ} \mathrm{C}$ was added $\mathrm{BBr}_{3}\left(1.7 \mathrm{~mL}, 1 \mathrm{M} \mathrm{BBr}_{3}\right.$ in dichloromethane) and the mixture was stirred under nitrogen for $30 \mathrm{~min}$ at $-10^{\circ} \mathrm{C}$ and then at room temperature for $30 \mathrm{~min}$. The reaction mixture was diluted with water, stirred for $20 \mathrm{~min}$ and extracted with chloroform. The organic extract was washed with brine and evaporated.

The resulting material was chromatographed over silica gel with hexane:ether (1:1) to give the compound 6 (130 mg, 46\%), m.p. $103-105^{\circ} \mathrm{C}$ (lit. $\left.{ }^{2} 106-107^{\circ} \mathrm{C}\right)$; I.R. $\mathrm{v}_{\max }\left(\mathrm{cm}^{-1}\right): 3392(\mathrm{OH})$, $1701(\mathrm{CO})$; MS (m/z): $190\left(\mathrm{M}^{+}\right)$(98\%), $175\left(\mathrm{M}^{+}-\mathrm{Me}\right)(42 \%), 147\left(\mathrm{M}^{+}-\mathrm{MeCHO}\right)(100 \%) ;{ }^{1} \mathrm{H}$ $\delta_{(\mathrm{ppm})}: 7.02(\mathrm{t}, 1 \mathrm{H}, \mathrm{J}=8 \mathrm{~Hz}),($ ArH at C-7), $6.72(\mathrm{~d}, 1 \mathrm{H}, \mathrm{J}=8 \mathrm{~Hz})(\mathrm{ArH}$ at C-8), $6.64(\mathrm{~d}, 1 \mathrm{H}, \mathrm{J}=8$ $\mathrm{Hz}$ ) (ArH at C-6), 5.75 (bs, 1H, OH), $2.80(\mathrm{~m}, 5 \mathrm{H}), 2.29(\mathrm{~s}, 3 \mathrm{H}, \mathrm{MeCO}), 1.74(\mathrm{~m}, 2 \mathrm{H}) ;{ }^{13} \mathrm{C} \delta_{(\mathrm{ppm})}$ : 212.1 (C-11), 153.5 (C-5), 136.7 (C-9), 126.3 (C-7), 122.5 (C-4), 121.2 (C-8), 112.2 (C-6), 47.6 (C-1), 31.1 (C-10), 28.2 (C-2), 24.9 (C-12), 22.6 (C-3). (Found: C, 75.98; H, 7.61; $\mathrm{C}_{12} \mathrm{H}_{14} \mathrm{O}_{2}$ requires $\mathrm{C}, 75.76 ; \mathrm{H}, 7.42)$.

\section{Acknowlegement}

The authors wish to thank Dr. N.R. Chatterjee, Pune, India and Lic. Po.S.Poon, N., Spain for providing us a reprint of the article of Reference 2.

\section{References}

1. Rao, A.V.R., Mehendale, A. R., Reddy, K.B. Tetrahedron Lett., 1982, 23, 2415.

2. Rao, A.V.R., Mehendale, A.R., Reddy, K.B. Indian J. Chem., 1984, 23B, 1154.

3. Yoshioka, K., Goto, G., Mabuchi, H., Hiraga, K., Miki, T. Chem. Pharm. Bull., 1975, 23, 3203.

4. Poos, G.I., Arth, G.E., Beyler, R.E., Sarett, L.H. J. Am. Chem. Soc., 1953, 75, 42. 